\title{
Facharztweiterbildung Pneumologie: Wie können wir sie verbessern?
}

\author{
Further Training for Medical Specialists in Respiratory Medicine: \\ How Can we Improve it?
}

Autor

Institut
O. Karg

Fortbildungsakademie Pneumologie der DGP
Bibliografie

Dol http://dx.doi.org/

10.1055/s-0034-1393052

Pneumologie 2015; 69: 515-520

(c) Georg Thieme Verlag KG

Stuttgart · New York

ISSN 0934-8387

\section{Korrespondenzadresse \\ Dr. Ortrud Karg}

Leiterin der

Fortbildungsakademie

Pneumologie der DGP

Zapfweg 11

81241 München

o.karg@t-online.de

\section{Zusammenfassung \\ $\nabla$}

Junge Ärzte in Deutschland bemängeln häufig ihre Facharztweiterbildung, hierbei vor allem fehlende Struktur und mangelhafte Rotationsplanung. Weiterbildungs- und Rotationspläne müssen mit dem Antrag einer Weiterbildungsermächtigung den Ärztekammern vorgelegt werden, aber sie werden bis jetzt nicht überprüft und nur partiell eingehalten. Weiterbilder befassen sich zu wenig mit Lehr- und Lerntheorie und Medizindidaktik. Evaluationen der Lernziele werden kaum durchgeführt, strukturierte Arbeitsplatz-bezogene Assessments sind eher die Ausnahme. Die Ursache liegt zumindest zum Teil in unserem System der Weiterbildung: Zusätzliche Ressourcen in den Weiterbildungsstätten sind nicht vorgesehen, der Einsatz für Weiterbildung wird nicht gegenfinanziert. Hinzu kommt, dass Lehre keinen hohen Stellenwert besitzt. Es setzt sich jedoch zunehmend die Erkenntnis durch, dass gute Weiterbildung zur Qualitätssicherung und auch für die Zukunftsperspektive einer Klinik erheblich beiträgt. Weiterbildung muss besser geplant, strukturiert und evaluiert werden. In die Lehre sollten neue Lehrmethoden mit aufgenommen werden. Die Deutsche Gesellschaft für Pneumologie und Beatmungsmedizin e.V. (DGP) möchte mit verschiedenen Angeboten dazu beitragen, Weiterbildung zu verbessern: beispielsweise mit Train-the-Trainer-Seminaren für die Weiterbilder, strukturierten Kursprogrammen für die Weiterbildungsassistenten, Assessmentangeboten wie dem HERMES (Harmonized Education in Respiratory Medicine for European Specialists)-Examen und Unterstützung bei der Akkreditierung zum „Respiratory Training Centre“ der ERS (European Respiratory Society) und EBAP (European Board for Accreditation in Pneumology).

\section{Abstract \\ $\nabla$}

Young physicians in Germany often criticize the advanced training programme, especially the lack of structure and the insufficient rotations. The Medical Association in each Bundesland/ federal state require to include a proposal for advanced training and rotation in a trainer's aplication for an educational license. However, there is no systematic scrutiny of these concepts and therefore the criteria stated outcomes are often only incompletely met. Trainers engage too little in training methods and medical didactics. They rarely evaluate learning outcomes, and structured assessments based on workplace are exceptions. The reasons are deeply rooted in Germany's education system: Resources for specialist training are not provided, and there is no funding for a commitment in continued medical education. In addition, teaching is not assigned a quantifiable value. However, during the last decade awareness has arisen that good training programmes are an important part of quality assurance and the validation of a hospital. Better planning, structuring and evaluation of training programmes is necessary. New learning methods should be incorporated in training programmes. The German Respiratory Society (DGP) wishes to contribute to the improvement of advanced training: for example with "train the trainer" seminars for teachers, with a structured educational course programme for the trainees, with assessments such as the HERMES (Harmonized Education in Respiratory Medicine for European Specialists) exam and with support for the accreditation as a Respiratory Training Centre of the ERS (European Respiratory Society) and EBAP (European Board for Accreditation in Pneumology). 


\section{Einleitung}

$\nabla$

„Wer evaluiert systematisch die Qualität der angebotenen Weiterbildungsmaßnahmen in seiner Klinik und wer führt eine strukturierte Abfrage über die Zufriedenheit der weiterzubildenden Ärzte mit der Facharztweiterbildung durch?“

Diese Fragen aus dem KTQ (Kooperation für Transparenz und Qualität im Gesundheitswesen)-Katalog [1] habe ich bei einer Veranstaltung Kollegen mit Weiterbildungsermächtigung für Pneumologie gestellt, die Antwort war ein klares „Niemand“. Dieses eindeutig negative Statement sollte Anlass zum Nachdenken über Verbesserungen geben. Verbesserung der Weiterbildung ist zudem eine der Hauptaufgaben der DGP-Fortbildungsakademie.

\section{Evaluation der Weiterbildung \\ $\nabla$}

„Die Facharztweiterbildung ist in Deutschland nicht mehr als ein Abfallprodukt ärztlicher Arbeit“. Diesen Vorwurf haben die „Jungen Internisten“ der Deutschen Gesellschaft für Innere Medizin (DGIM) und das „Junge Forum“ des Berufsverbandes Deutscher Internisten (BDI) erhoben [2]. Sie beziehen sich auf das Ergebnis einer Umfrage, die sie Ende 2014 durchgeführt und an der sich knapp 1700 Ärzte in Weiterbildung beteiligt haben. Bemängelt wird vor allem die fehlende Struktur der Weiterbildung:

- Nur $17 \%$ der Befragten gaben an, dass sie zu Beginn ihrer Weiterbildung wussten, wann und in welche Bereiche sie rotieren werden, $83 \%$ hatten keinen strukturierten Ausbildungsplan.

- Weiterbildungsgespräche fänden in $23 \%$ gar nicht, in $45 \%$ nur unregelmäßig und lediglich in $32 \%$ wie gefordert statt. Als hilfreich empfunden werden diese Gespräche, die eigentlich aufdecken sollen, wie die Weiterbildung tatsächlich läuft, nur von $28 \%$. „Sie werden von den Vorgesetzten eher als bürokratische Belastung angesehen“, meist fänden sie daher zwischen Tür und Angel statt und erst dann, wenn es die Unterschrift für den Weiterbildungsabschnitt gäbe.

- Mit dem Berufsalltag zufrieden seien lediglich $40 \%$. Als häufigste Gründe für Unzufriedenheit wird schlechte Weiterbildung (14,9\%), hoher Zeitdruck (14,8\%) und hohe Arbeitsbelastung (14,1\%) angegeben.

- Die Qualität der Patientenversorgung bewerten 86\% als gefährdet, Hauptgründe hierfür seien hohe Arbeitsverdichtung (33\%), zahlreiche nicht ärztliche Tätigkeiten (27\%), unzureichende Supervision (20\%), aber auch mangelnde Fort- und Weiterbildung (17,1\%).

- Auch der ökonomische Druck wird als zu hoch empfunden: 90\% gaben an, dass ökonomische Erwägungen bei ärztlichfachlichen Entscheidungen eine Rolle spielen würden [3].

Die Reaktion von Dr. Thomas Schröter, Vorstandsmitglied des BDI, auf dieses Umfrageergebnis lautete: „Jeder der Weiterbildung macht, muss sich verpflichten, eine strukturierte Weiterbildung zu machen“ [4].

Die Bundesärztekammer (BÄK) hat gemeinsam mit den Landesärztekammern bereits in den Jahren 2009 und 2011 Umfragen zur Evaluation der Weiterbildung durchgeführt, in 2011 hatten sich fast 30000 Ärzte in Weiterbildung daran beteiligt. Die Kernaussagen der Erhebung beziehen sich auf acht Fragenkomplexe: Globalbeurteilung, Vermittlung von Fachkompetenz, Lernkultur, Führungskultur, Kultur zur Fehlervermeidung, Entscheidungskultur, Betriebskultur, Wissenschaftlich begründete Medizin.
Die durchschnittliche Bewertung nach Schulnoten lag damals zwischen 2,09 und 3,25 (Mittelwert 2,4), kann somit als durchschnittliche Zufriedenheit mit der Weiterbildung interpretiert werden. Lediglich der Komplex „Wissenschaftlich begründete Medizin“ setzt sich von den anderen Ergebnissen mit einer deutlich schlechteren Note $(3,25)$ ab $[5,6]$.

Aber auch in dieser Umfrage wurde bemängelt, dass lediglich in $58 \%$ ein strukturierter Weiterbildungsplan zur Kenntnis gegeben wurde, $32 \%$ gaben an, dass gar keine - auch keine mündlichen Lern- und Weiterbildungsziele vereinbart worden sind. Überbordende Bürokratie würde Patientenversorgung und Weiterbildung gleichermaßen behindern.

\section{Lern-|Lehrmethoden \\ $\nabla$}

Die Zahlenangaben über die Zufriedenheit mit der Weiterbildung schwanken in den verschiedenen Umfragen erheblich. Dennoch bleibt festzustellen: Junge Ärzte wünschen sich Änderungen in der Weiterbildung. Das Junge Forum des BDI hat z. B. eine Checkliste „WBO-freundliches Krankenhaus erstellt, nach der Kollegen bei ihren Vorstellungsgesprächen die ihnen auf den Nägeln brennenden Punkte abfragen sollen [7]. Weiterbilder sollten sich diese Liste ebenfalls ansehen. Sie zeigt, was jungen Ärzten wichtig ist. An dem alten Vorwurf, dass die junge Generation nicht mehr leistungsbereit sei, stimmt nur eins: Er ist alt. Aber das Lernumfeld, in dem diese Generation aufgewachsen ist, hat sich erheblich gewandelt: Wir, die Nachkriegsgeneration, haben unser theoretisches Wissen überwiegend noch aus Zeitschriften und Büchern in Bibliotheken bezogen oder Experten befragt, Vorträge und Vorlesungen wurden mit Dias oder Overheadfolien gestaltet. Die folgende sog. Generation X lernte bereits mit e-books und e-journals, die im Internet zur Verfügung standen, es begann die Ära der Powerpoint-Präsentationen. Die jetzige sog. Generation Y bezieht ihr Wissen überwiegend aus sozialen Medien und Netzwerken, lernt multimedial auf unterschiedliche Weise. Die neuen Lernmethoden sind u.a. Simulationen, virtuelles oder spielerisches Lernen, YouTube-Instruktionen. Diese Generation wünscht sich eine strukturiertere Form der Weiterbildung sowie ein kontinuierliches Feedback, was uns wiederum eher kränkte [8]. Diese neuen Lernmethoden muss auch die Fachgesellschaft in ihrem Fortbildungsangebot berücksichtigen und sinnvoll traditionelle und neue technologiegestützte didaktische Bildungskonzepte kombiniert einsetzen.

Im HERMES-Akkreditierungskatalog für Respiratory Training Centers von ERS/EBAP werden als Lehr- und Lernmethoden gefordert [9]:

- Unterricht am Krankenbett/auf der Station, bei Operationen oder Eingriffen, in der Ambulanz

- Fallbezogene Diskussion

- Bewertung von Arztbriefen mit Darstellung der Beurteilungskriterien

- Patientenvorstellung im interdisziplinärem Team (sog. Grand rounds: Abteilungsmeeting, Lehrseminar)

- Morgendlicher Visitenrundgang

- Lernen mit Kollegen

- Autodidaktisches Lernen

- E-Learning

Diese und ähnliche Methoden werden auch in anderen Weiterbildungsprogrammen, z.B. von Großbritannien [10] aufgeführt. Sie sind auch nur teilweise neu, entsprechen ansonsten dem gelebten Alltag. 


\section{Evaluationsmethoden am Arbeitsplatz}

Aus dem Qualitätsmanagement haben wir gelernt, dass wir alle Maßnahmen im Hinblick auf ihre Effizienz evaluieren müssen. Prüfungen - eine der ältesten Methoden von Qualitätsmanagement - sind nun wirklich nichts Neues, aber wir sollten diese ebenfalls strukturiert durchführen und nur zusammen mit konstruktivem Feedback. Letzteres erhöht Motivation und Anstrengung. Wenn Evaluationen und Feedbackgespräche zur Routine werden, nimmt auch die emotionale Anspannung in der Prüfungssituation ab. In der Schweiz sind z.B. mindestens vier Arbeitsplatz-basierte Assessments pro Jahr vorgeschrieben [11]. International gängige Assessmentmethoden, wie sie u.a. im HERMES-Akkreditierungskatalog angegeben werden, sind [9]:

$\checkmark$ Audit

- Erörterung am Fallbeispiel

- Direkte Beobachtung praktisch-klinischer Fertigkeiten (DOPS)

- Mini-Clinical Evaluation Exercise

(Mini-CEX = Praktische Prüfung und Feedback)

- Beurteilung durch verschiedene Kollegen (Multi-Source Feedback)

- Objective structured clinical examination (OSCE)=Prüfung klinischer Kompetenz anhand unterschiedlicher Aufgaben bei standardisierten Patienten und mit festen Zeitvorgaben

- Mündliche Prüfung

- Andere Optionen wie z.B. Simulation standardisierter Patienten

Zwei einfach durchzuführende, international anerkannte Arbeitsplatz-basierte Assessments sind DOPS (Directly Observed Practical Skills) und Mini-CEX (Clinical Evaluation Exercise): Praktische ärztliche Kompetenzen werden im klinischen Alltag strukturiert beobachtet und evaluiert [11]. Ähnliche Methoden wird jeder Weiterbilder anwenden, aber eben häufig unstrukturiert und ohne Dokumentation. Im Fokus von DOPS stehen vor allem Interventionen wie Ultraschall, Pleurapunktion, Drainage, Bronchoskopie, im Fokus von Mini-CEX vor allem Anamnese, Klinische Untersuchung, Aufklärungsgespräche, Befundbesprechungen, Beratung [12]. Es erfolgt sowohl eine Selbst- als auch Fremdbeurteilung. Beurteilungskriterien sind z.B. Aufklärung, Vorbereitung, Durchführung, Hygiene, Kommunikation. Entsprechende Dokumentationsbögen stellt die DGP auf der Homepage zur Verfügung [13]. Dabei ist ein konstruktives Feedback wichtig: Was war gut, was kann verbessert werden [14]? Daraus ergeben sich dann wiederum gemeinsam formulierte neue Lernziele. Aufwändiger ist dagegen das Assessment „Multi-Source Feedback“.

\section{Kompetenz und Performanz}

$\nabla$

Diese Begriffe klingen zunächst nach viel abstrakter Theorie. Aber die Benennung und Beschreibung der einzelnen Lehr- und Evaluationsmethoden zwingt uns, unsere eigenen Methoden zu überdenken und erforderlichenfalls zu verbessern. Lehren und Lernen sollten wie andere Prozesse einer ständigen Professionalisierung bzw. einem Qualitätszyklus unterliegen. So sollten angestrebte Lernziele das für die klinische Praxis relevante Wissen, die praktischen Fertigkeiten und das persönliche Verhalten reflektieren. Persönliches Verhalten (behaviour/attitudes) bedeutet dabei sowohl das Verhalten im Umgang mit Patienten und deren Angehörigen als auch das Verhalten im interdisziplinären Team. Dies sind die Eckpunkte für klinische Kompetenz, die für die professionelle Durchführung von ärztlichen Aufgaben benötigt wird. Klinische Kompetenz kann nach Miller als Lernpyramide in verschiedenen Ebenen dargestellt werden [15]: Auf Ebene eins hat man Fachwissen erworben (knows), auf Ebene zwei Handlungswissen (knowshow), die Ebene drei bedeutet eine Prüfungssimulation (shows), Ebene vier selbstständige Durchführung (does). Kompetenz bedeutet somit die (latente) Fähigkeit, eine bestimmte Aufgabe ausführen zu können, Performanz bedeutet dagegen die tatsächliche Ausführung - „man muss es nicht nur können, sondern auch zeigen“. Auch wenn dieses Modell viel kritisiert wird, benennen die meisten Aus- und Weiterbildungsprogramme diese oder vergleichbare Kompetenzebenen. Dies gilt auch für die im Abstimmungsprozess befindliche Änderung der Musterweiterbildungsordnung der BÄK ebenso wie für den im Sommer 2015 beschlossenen Nationalen Kompetenzbasierten Lernzielkatalog Medizin (NKLM).

Weiterbilder müssen Kompetenz und Performanz ihrer Ärzte in Weiterbildung fortentwickeln. Dabei kann man Performanz in kleine Schritte zerlegen, d.h. man kann festlegen, was man zu welchem Zeitpunkt jungen Ärzten an selbstständiger Tätigkeit ohne Aufsicht zutraut. Diese bezeichnet man als Entrustable Professional Activity (EPA): Entrustable professional activities represent the outcomes of training, the activities that society and professional peers can expect fellowship graduates to be able to perform unsupervised [16].

Das American College of Chest Physicians (ACCP) hat in 2014 einen derartigen Katalog von EPA's für die Pneumologie und Intensivmedizin erarbeitet [16]. Performanz kann man in einem EPA-Bewertungsborgen erfassen, die einfachste Form ist wiederum DOPS oder Mini-CEX.

Die Wege zur Performanz haben sich in den letzten Jahrzehnten geändert: Stand bei unserer Weiterbildung z.B. Lernen durch Handeln (learning by doing) im Vordergrund, so ist es heute eher Lernen am Simulator (Simulationsmodell oder Computer). Learning by doing lassen sich heute Patienten kaum noch gefallen, wird gesellschaftlich nicht mehr akzeptiert.

\section{Weiterbildungsorganisation \\ $\nabla$}

Ein System wie in Deutschland, bei dem Weiterbildung tatsächlich ein Nebenprodukt der täglichen ärztlichen Arbeit ist, gibt es international selten. Dennoch sollte man unser System nicht schlecht reden. Vorteile sind garantierte Mobilität, die Möglichkeit der freien Facharztwahl und große Freiheit des Einzelnen. Eine Steuerung findet zumindest bisher nicht statt. In Deutschland werden Arbeitsverträge, keine Weiterbildungsverträge abgeschlossen. Weiterbildung ist ärztliche Tätigkeit, gehört nicht mehr zur Ausbildung. In Frankreich dagegen ist ein Arzt in Weiterbildung eher Student mit entsprechend schlechter Bezahlung. Ob man in anderen Ländern die Fachrichtung seiner Wahl erhält, hängt oft nicht von Qualität, Qualifikation und Ort, sondern von Glück und Verteilungsquoten ab. Es wird jedoch bei uns innerhalb der Weiterbildung viel dem Zufall überlassen. Andere Systeme sind wesentlich reglementierter, ein Vorteil davon ist jedoch die bessere Strukturierung [17]. Wir - zumindest die Nachkriegsgeneration - empfinden dies wiederum als „Verschulung“. Man könnte aber Weiterbildungsverträge mit klarer Strukturierung neben den arbeitsrechtlichen Beschäftigungsverträgen schließen. Ob dies bei uns schon praktiziert wird, ist mir nicht bekannt. Ein großer Nachteil unseres Systems sind der Mangel an Zeit und fehlende personelle Ressourcen, da keine Gegenfinanzierung erfolgt. In Großbritannien z.B. ist die Weiterbildung 
sehr strukturiert und wird stark kontrolliert. Junge Ärzte werden dort behutsam an ihre Aufgaben herangeführt, Kliniken erhalten entsprechende Budgets und Zeit für die Weiterbildung.

Die meisten Fachärzte arbeiten nach der Weiterbildung in ambulanten Praxen. Um für diese Tätigkeit besser vorbereitet zu sein, sollte ein Teil der Weiterbildung unbedingt in ambulanten Lehrpraxen stattfinden. Die organisatorischen und finanziellen Bedingungen hierfür müssen geschaffen werden.

\section{Weiterbildungsermächtigungen}

Die Inhalte der Musterweiterbildungsordnung der BÄK sind von den Landesärztekammern fast einheitlich übernommen und anerkannt. Im Gegensatz dazu sind die Kriterien, nach denen eine Weiterbildungsbefugnis vergeben wird, sehr undifferenziert ausgestaltet. Dies führt zu einer heterogenen Weiterbildungsqualität und damit zu einer Chancenungleichheit für Weiterzubildende. DGIM und BDI haben in einer Stellungnahme 2012 gefordert, dass die Kriterien zur Befugnis zur Weiterbildung um die Anforderung erweitert werden, strukturierte Weiterbildungspläne mit Vereinbarung von Lern- und Weiterbildungszielen als Standard zu etablieren [18]:

- Die Entwicklung dieser Pläne soll von den Landesärztekammern geprüft werden, um eine einheitlich gute Qualität der Weiterbildung zu garantieren.

- Die Überprüfung und die Erteilung der Weiterbildungsbefugnisse müssten nach transparenten Kriterien erfolgen und von den Landesärztekammern offen gelegt werden.

- Unnötige Anforderungen der Weiterbildungsordnung und überhöhte Richtzahlen sollen durch effizientere Strukturen der Weiterbildung und verbesserte Didaktik in den Weiterbildungsstätten ersetzt werden.

- Verbindlich geplante, fachübergreifende Weiterbildungen und Rotationen könnten diese Strukturen unterstützen, einzelne Weiterbildungsstätten entlasten und die Qualität der Weiterbildung erhöhen.

Um eine Weiterbildungsermächtigung zu erhalten, ist die Vorlage von Weiterbildungsplänen bei den Kammern mit Angaben von Rotationen seit langer Zeit Vorschrift. Diese Pläne werden auch von vielen Kliniken auf ihren Websites veröffentlicht. Aber sie werden nicht überprüft, sind damit oft nur Makulatur. In anderen Ländern (z.B. Schweiz) finden dagegen Visitationen zur Evaluation statt.

Auch unsere Facharztprüfungen sind wenig strukturiert. Für die mündliche Prüfung, die auf der Diskussion mehrerer Patientendossiers basiert, existieren z.B. keine vordefinierten Minimalkriterien für das Bestehen.

\section{Struktur von Weiterbildungsplänen \\ $\nabla$}

Wie sollte ein strukturierter Weiterbildungsplan nun aussehen?

Er ist ein Weiterbildungskonzept, das die Vermittlung der Lerninhalte zeitlich und inhaltlich strukturiert dokumentiert. Er muss realistisch und nachvollziehbar das Weiterbildungsangebot und vor allem die Ziele definieren, die ein Arzt in Weiterbildung z. B. während eines Jahres erreichen kann [19]. Die Zahl der Weiterbildungsstellen, die in Deutschland nicht festgelegt ist, sollte in einem ausgewogenen Verhältnis zur Menge der für die Weiterbildung verfügbaren Patienten stehen. Auch sollte ein angemes- senes Verhältnis zwischen der Anzahl weiterzubildender Personen und der Anzahl der Weiterbilder (Tutoren) festgelegt werden [20]. Neben der Angabe von Rotationsbereichen (z.B. thorakale Onkologie, Infektiologie, Schlaf- und Beatmungsmedizin) sollen Rotationszeiten und vor allem Lerninhalte und Lernziele der einzelnen Bereiche angegeben werden.

Es sollte auch festgehalten werden, in welcher Zeitschiene Lerninhalte aus Funktionsbereichen (z. B. Lungenfunktion, Bronchoskopie, thorakaler Ultraschall), in die meist keine Rotation erfolgt, parallel zu den Lerninhalten der jeweiligen Station, vermittelt werden.

Zum Weiterbildungsangebot gehören auch die in der Abteilung stattfindenden Konferenzen und Veranstaltungen, sie sind Teil des Lehrprogramms.

In den publizierten Weiterbildungsplänen deutscher Kliniken werden Evaluationsmethoden und -häufigkeiten praktisch nicht erwähnt: Wie werden die gesetzten Lernziele überprüft, wie häufig z.B. Weiterbildungsgespräche durchgeführt und wie dokumentiert? Art und Häufigkeit der Assessments sollten angegeben werden. Das Führen von Logbüchern ist zwar Pflicht, aber teilweise werden sie noch immer erst am Ende der gesamten Weiterbildung ausgefüllt. Zur Erleichterung der Dokumentation werden entsprechende elektronische Systeme angeboten.

\section{Unterstützung durch die Fachgesellschaft}

Wie können wir als Fachgesellschaft Ihre Bemühungen für eine bessere Weiterbildung unterstützen?

1. Verbesserung des medizindidaktischen Wissens der Weiterbilder z.B. durch Train-the-Trainer-Seminare und andere Veranstaltungen.

Nach dem Modell von „CanMEDS“ [21], dem Weiterbildungsprogramm von Kanada, das zwischenzeitlich von vielen Ländern übernommen worden ist, hat ein Arzt sieben Rollenfunktionen (Medizinexperte, Gelehrter, Gesundheitsberater, Kommunikator, Verantwortungsträger, Teammitglied, Professionell Handelnder) auszuüben, darunter die des Gelehrten oder „Lehrenden“. Aber wer hat hierin Kompetenzen erworben, wer ist z.B. wirklich informiert über neue Lehr- und Lernmethoden mit dem Einsatz digitaler Lerntechnologien und -medien? Wer kennt die verschiedenen Assessmentinstrumente und wendet sie auch in der Praxis an? Den „Master of Medical Education“ haben nur wenige Universitätskollegen erworben, die sich wiederum dem Vorwurf ausgesetzt sehen, hauptsächlich Theoretiker zu sein. Zweifellos gibt es - jedoch eher selten - naturbegabte Lehrer, weniger Begabte müssen sich zumindest Basiskompetenzen erwerben in generellen Prinzipien von Aus- und Weiterbildung sowie didaktischem Wissen [22].

Hier kann die Fachgesellschaft u.a. durch das Angebot entsprechender Seminare, Postgraduiertenkurse oder Symposien die Lücke schließen, sodass der Weiterbilder auch ein guter,„Lernbegleiter" wird.

Bisher gibt es jedoch keine relevante Nachfrage nach derartigen Veranstaltungen, fachliche Fortbildung in Sachen Lehre hat immer noch keinen Stellenwert. Eine Nachfrage meinerseits bei Mitarbeitern der Kompetenzbereiche Hochschuldidaktik und eLearning des Dieter Scheffner Fachzentrums der Charité, wie oft sie denn von medizinischen Fachgesellschaften zu Veranstaltungen über Medizindidaktik eingeladen werden, ergab die Antwort, dass dies wirklich nur sehr selten der Fall sei. 
2. Angebot eines überregional oder regional organisierten Kursprogrammes

Die DGP kann die Weiterbildungsangebote der Kliniken mit einem strukturierten Kursprogramm für Weiterzubildende unterstützen. Lerninhalte werden nach einem fixen Lernzielkatalog vermittelt. Ein derartiges Programm ginge weit über die bisher angebotenen Vorbereitungs-(State of the Art) Kurse für die Facharztprüfung hinaus. In Dreijahreszyklen könnten alle wesentlichen Themen in mehrtägigen, z. B. zweimal jährlich stattfindenden Seminaren abgehandelt werden. Voraussetzung wäre allerdings, dass die Klinikträger den Weiterzubildenden den Besuch ermöglichen und die Weiterbilder dies zur Pflicht machen und sich selbst bei diesen Veranstaltungen als Lehrende einbringen. Das würde wahrscheinlich mehr bewirken als der sog. Crashkurs am Ende der Weiterbildungszeit zur Prüfungsvorbereitung. Ich möchte den „State of the Art“-Kurs in keiner Weise abwerten. Die Lerninhalte sind am HERMESCurriculum ausgerichtet, die Bewertung durch die Teilnehmer ist gut. Den Initiatoren, Organisatoren und Referenten sei an dieser Stelle Dank ausgesprochen. Meines Erachtens müssen wir jedoch unsere Aktivitäten in Bezug auf Lehrveranstaltungen erhöhen. In sechs Seminaren wäre dann auch Zeit für Simulationen, Falldiskussionen und persönlichen Austausch an Stelle von reinen Fachvorträgen über zehn Stunden täglich. In manchen Abteilungen werden ja durchaus entsprechende einzelne Veranstaltungen angeboten, oft aber nur über das Thema, für das die Klinik ein gewisses Renommee besitzt. Selten geschieht dies jedoch strukturiert mit fester Themenabfolge und manchmal auch in Konkurrenz zueinander. Dabei spielt dann sogar der Klinik-Veranstaltungsort eine Imagerolle. Eine Abteilung allein kann den erforderlichen Aufwand für ein strukturiertes umfassendes Programm nicht stemmen, hier muss die Vernetzung untereinander das Konzept stützen. Denkbar wären regionale Verbünde mit Beteiligung aller Weiterbilder. Die Fachgesellschaft, v.a. auch die Regionalgesellschaften könnten bei der Organisation dieser regionalen Veranstaltungen helfen, würden evtl. auch die Konkurrenzsituation entschärfen.

Nachfragen haben jedoch ergeben, dass zumindest bisher die Notwendigkeit für ein aufwendiges Weiterbildungskursprogramm nach Lernzielkatalog nicht gesehen wird.

Für Bereiche der Weiterbildung, die in vielen Abteilungen stationär nur noch schwierig zu erbringen sind, wie z. B. die Allergologie, haben wir eine moduläre Weiterbildung entworfen: Hier werden die theoretischen Kenntnisse ebenfalls in einem Kurs erworben, die in der Weiterbildungsordnung geforderten praktischen Nachweise müssen während Hospitationen oder Rotationen in einer Praxis abgeleistet werden. Wir haben das Konzept der Bayerischen Landesärztekammer zur Prüfung vorgelegt, warten noch auf eine Stellungnahme. Bei derartigen Modellen darf eine Rotation in die Praxis für den weiterzubildenden Arzt keine Nachteile, z.B. in der Vergütung, mit sich bringen.

3. Assessmentangebot: HERMES-Examen

Zur Überprüfung des theoretischen Fachwissens (von Weiterbildern und Weiterzubildenden) hat die DGP in Kooperation mit der ERS in 2015 erstmals das HERMES-Examen angeboten. Es reiht sich ein in die Assessmentmethoden. 90 MultipleChoice-Fragen über die im HERMES Core Syllabus [23] bzw. Curriculum [24] aufgeführten Lerninhalte müssen in drei Stunden beantwortet werden. Es haben 17 Kollegen, davon 11 Ärzte in Weiterbildung, teilgenommen. Eine kleine Umfrage unsererseits hat ergeben, dass die Mehrzahl der Teilnehmer das Examen als gute Methode zur Wissensüberprüfung einschätzte und gerne auch wieder teilnehmen würde. Wir werden das Examen auch am Kongress in 2016 anbieten (Mittwoch, 2. März 2016), hoffen auch im Hinblick auf den Aufwand auf eine etwas größere Teilnehmerzahl und appellieren an die Weiterbilder, ihre Mitarbeiter zur Teilnahme an dem Test zu motivieren und durch eigene Teilnahme mit gutem Beispiel voranzugehen.

4. Förderung der Akkreditierung als Respiratory Training Center Zum HERMES-Programm der ERS zählen neben dem Weiterbildungscurriculum das HERMES-Examen und die Akkreditierung von Respiratory Training Centers. Die pneumologische Abteilung im Emil-von-Behring-Krankenhaus in Berlin (Lungenklinik Heckeshorn) wurde als erste Klinik in Europa von der ERS/EBAP akkreditiert. Die DGP unterstützt dieses Akkreditierungsverfahren durch Mitarbeit im Akkreditierungskomitee und wünscht sich eine rege Teilnahme.

Warum sollte man sich so einem Akkreditierungsprozess unterziehen, was kann er bewirken? Wie bei allen Zertifikaten wird die Klinik bzw. der Weiterbilder gezwungen, seine Prozesse - in dem Fall Weiterbildungsprozesse - zu dokumentieren, zu reflektieren und zu verbessern, d.h., es wird der bekannte Qualitätsmanagementprozess in Gang gesetzt. Bei der Vorbereitung der erforderlichen Unterlagen werden Mängel auffällig, die Audits der externen Reviewer helfen beim Aufbau entsprechender Strukturen.

Voraussetzungen für eine Akkreditierung sind [9]:

- Beschreibung eines einzelnen Training Centers oder eines Netzwerks (Verbund mehrerer Abteilungen mit Kooperationsvertrag)

- Strukturvoraussetzungen des Training Centers für ein Weiterbildungsprogramm

- Klinische Expertise (gesamtes pneumologisches Spektrum, ca. 300 stationäre Patienten/Jahr/Trainee, ca. 150 Erst- und 400 Kontroll-Untersuchungen ambulante Patienten/Jahr/ Trainee, Führung eines Logbuchs $\rightarrow$ regelmäßig abgezeichnet, Verfügbarkeit erfahrener Ansprechpartner für jede Neuaufnahme, Verfügbarkeit fachfremder Konsile in akzeptabler Zeit)

> Weiterbildungs-Expertise (Trainees entwickeln klinische Erfahrung durch Erwerb von Wissen und klinischer Praxis, Kompetenz-basiertes Weiterbildungsprogramm, Anwendung aller moderner Lehrmethoden, Anwendung geläufiger Assessmentmethoden)

- Wissenschaftliche Expertise (Training Center muss Publikationen vorweisen, Training Center muss Trainees Forschung ermöglichen - jeder Trainee sollte mindestens ein Poster auf einem internationalen Kongress präsentiert haben)

- Infrastruktur (Arbeitsräume, Ausstattung, Internet-Zugang, Zugang zu Literatur, Einhaltung der Arbeitszeit - hierzu erfolgt Befragung der Trainees, Bereitstellung von Arztbriefen, Röntgen-, Labor- und Patho-Befunden, Unterstützung der ärztlichen Tätigkeit durch Assistenzpersonal, z. B. StationssekretärIn)

> Organisation Weiterbildungsprogramm (Qualifikationen der Weiterbilder - sollten ein HERMES-Diplom erworben haben, methodische Fortbildung)

- Anforderungen an spezifische Einrichtungen (Radiologie, Lungenfunktion, Endoskopie, Therapeutische Interventionen wie LTOT, NIV, Intensivmedizin, Schlafmedizin, Palliativmedizin, Raucherentwöhnungsprogramm u.a. mit Kennzahlen) 
Das erhaltene Qualitätssiegel bedeutet eine Reputation für eine gute Weiterbildung und Attraktivität für junge Ärzte (siehe Checkliste für WBO-freundliches Krankenhaus), zieht eine höhere Außenwirkung nach sich und ist für den Träger evtl. ein Anreiz zur Ausstattung mit ausreichend Personal, Räumlichkeiten und technischer Ausrüstung sowie Angebot des vollen Spektrums diagnostischer und therapeutischer Techniken entsprechend dem Weiterbildungscurriculum.

Unser nationales Weiterbildungssystem können wir nicht ändern. Durch Teilnahme möglichst vieler Kliniken an diesem Akkreditierungsprozess könnten wir aber zeigen, dass die Facharztweiterbildung zumindest in der Pneumologie eben nicht nur ein Nebenprodukt täglicher ärztlicher Arbeit ist, sondern dass wir uns nachhaltig dafür einsetzen.

\section{Schlussfolgerung}

Im Klinikalltag steht Weiterbildung zur Zeit noch hinten an, Patientenversorgung und Wirtschaftlichkeit der Klinik haben Priorität. Dies müssen wir ändern: Weiterbildung dient der der Qualitätssicherung und beruflichen Personalentwicklung, verbessert Patientenversorgung und -sicherheit. Wird dies erkannt, wird auch Weiterbildung in den Fokus des Interesses von Klinikträgern rücken.

Wir appellieren an alle Weiterbilder, sich intensiv für eine Verbesserung der Weiterbildung einzusetzen. Die Fachgesellschaft will Sie hierbei unterstützen. Falls Sie hierzu selbst Vorschläge haben, schreiben Sie diese bitte an die Fortbildungsakademie der DGP.

\section{Interessenkonflikt}

Die Autorin gibt an, dass kein Interessenkonflikt besteht.

\section{Literatur}

1 KTQ-GmbH. KTQ-Manual inkl. KTQ-Katalog 2009/Version 2 für das Krankenhaus. Berlin: Fachverlag Matthias Grimm; 2009

2 Höhl R. Dringende Reform der Weiterbildung nötig Ärztezeitung 20. 04. 2015

3 Raspe M, Müller-Marbach A, Schneider $M$, Schulte $K$ für Die Jungen Internisten der DGIM und das Junge Forum des BDI Deutschlandweite Befragung von WeiterbildungsassistentInnen in der Inneren Medizin 121. DGIM-Kongress Mannheim: 18. 04. 2015: www.bdi.de/fileadmin/ PDF/Junges_Forum/Vorstellung_Umfrage_DGIM_Kongress.pdf [Zugriff 05.08.2015]

4 Klein F. Die internistische Weiterbildung fällt durch Ärztezeitung 21. 04.2015
5 Bundesrapport der BÄK: Ergebnisse der Evaluation der Weiterbildung 2. Befragungsrunde 2011. www.evaluation-weiterbildung.de/ data/Bundesreport2011.pdf [Zugriff 05.08.2015]

6 BÄK. Ground Hintergrundinformationen für Journalisten - Pressestelle der deutschen Ärzteschaft 113. Deutscher Ärztetag in Dresden. www. bundesaerztekammer.de/fileadmin/user-upload/downloads/BAeK GROUND_Evaluation_der_Weiterbildung_web.pdf[Zugriff 05.08.2015]

7 Checkliste WBO-freundliches Krankenhaus. www.bdi.de/organisation/junges-forum/weiterbildung [Zugriff 05.08.2015]

8 Busari JO. The discourse of generational segmentation and the implications for postgraduate medical education. Perspect Med Educ 2013; 2: $340-348$

9 Loddenkemper R, Séverin T, Mitchell $S$ et al. Adult HERMES: criteria for accreditation of ERS european training centres in adult respiratory medicine. Breathe 2010; 7: 170-188

10 Joint Royal Colleges of Physicians Training Board. Specialty Training Curriculum for Respiratory Medicine. www.jrcptb.org.uk/sites/ default/files/2010 Respiratory (ammendments 2014).pdf [Zugriff 05.08.2015]

11 Schweizerisches Institut für ärztliche Weiter- und Fortbildung. Informationen zum Arbeitsplatz-basierten Assessment. www.fmh.ch/ files/pdf16/aba_infoblatt_d.pdf [Zugriff 05.08.2015]

12 Schweizerisches Institut für ärztliche Weiter- und Fortbildung. Vorschläge für Interventionen im Rahmen des Arbeitsplatzbasierten Assessments. www.fmh.ch/files/pdf15/pneumo_list_2014_de.pdf [Zugriff 05.08.2015]

13 Deutsche Gesellschaft für Pneumologie. Fortbildungsakademie/Curricula.www.pneumologie.de/780.0.html Zugriff 05.08.2015]

14 Schweizerisches Institut für ärztliche Weiter- und Fortbildung. Arbeitsplatz-basiertes Assessment. www.fmh.ch/files/pdf16/assessmentbogen_kombi_gen_JB_d_20131010.pdf [Zugriff 05.08.2015]

15 Miller GE. The assessment of clinical skills/competence/performance. Acad Med 1990; 65: 63-67

16 Fessler HE, Addrizzo-Harris D, Beck JM et al. Entrustable professional activities and curricular milestones for fellowship training in pulmonary and critical care medicine. Report of a multisociety working group. Chest 2014; 146: 813-834

17 Retzlaff L. Im Gespräch mit Mohrhardt C und Hammerschmidt A, Vertretern der European Junior Doctors. Was im Ausland besser ist - und was nicht. Marburger Bund Zeitung 24. 04. 2015; 6

18 Hallek $M$, Schröter $T$ für die DGIM und den BDI Die Zukunft der internistischen Weiterbildung in Deutschland. Dtsch Med Wochenschr 2012; 137: 2591 - 2594

19 Schweizerisches Institut für ärztliche Weiter- und Fortbildung. Weiterbildungsprogramm für Facharzt für Pneumologie. www.fmh.ch/ bildung-swif/fuer-leiter-wb-staetten/weiterbildungsstaetten/facharzttitel.html [Zugriff 05.08.2015]

20 Schweizerisches Institut für ärztliche Weiter- und Fortbildung. Muster-Raster für die Weiterbildungskonzepte gemäß Art. 41 WBO. www.fmh.ch/files/pdf15/130402_Muster-WB-Raster_vs_siwf_genehmigt_d.pdf [Zugriff 05.08.2015]

21 Frank JR. Office of Education The Royal College of Physicians and Surgeons of Canada. The CanMEDS 2005 Physician Competency Framework.http://rcpsc.medical.org [Zugriff 05.08.2015]

22 Busari JO. Current insights and potential benefits for effective teaching and learning in respiratory medicine. Breathe 2012; 9: 15-24

23 Loddenkemper R, Haslam PL, Séverin T et al. HERMES: a European Core Syllabus in Respiratory Medicine. Breathe 2006; 3: 59-70

24 Loddenkemper R, Haslam PL, Séverin T et al. European curriculum recommendations for training in adult respiratory medicine: $2^{\text {nd }}$ report of the HERMES Task Force. Breathe 2008; 5: 80-93 